\title{
Bloom syndrome in a Mexican American family with rhabdomyosarcoma: evidence of a Mexican founder mutation
}

\author{
Erin H. Sybouts, ${ }^{1,2,11}$ Adam D. Brown, ${ }^{1,2,8,11}$ Maria G. Falcon-Cantrill, ${ }^{3,9,11}$ \\ Martha H. Thomas, ${ }^{1,10}$ Thomas DeNapoli, ${ }^{4,5}$ Julie Voeller, ${ }^{5,6}$ Yidong Chen, ${ }^{1,7}$ \\ Gail E. Tomlinson, ${ }^{1,3}$ and Alexander J.R. Bishop ${ }^{1,2}$ \\ ${ }^{1}$ Greehey Children's Cancer Research Institute, ${ }^{2}$ Departments of Cell Systems and Anatomy, ${ }^{3}$ Pediatrics, UT \\ Health San Antonio, San Antonio, Texas 78229, USA; ${ }^{4}$ CHRISTUS Health, San Antonio, Texas 78251, USA; \\ ${ }^{5}$ Children's Hospital of San Antonio, San Antonio, Texas 78207, USA; ${ }^{6}$ Baylor College of Medicine, Houston, \\ Texas 77030, USA; ${ }^{7}$ Population Health Sciences, UT Health San Antonio, San Antonio, Texas 78229, USA
}

Corresponding author: tomlinsong@uthscsa.edu

(c) 2021 Sybouts et al. This article is distributed under the terms of the Creative Commons

Attribution-NonCommercial License, which permits reuse and redistribution, except for commercial purposes, provided that the original author and source are credited.

Ontology terms: embryonal rhabdomyosarcoma; moderately short stature;

rhabdomyosarcoma

Published by Cold Spring Harbor Laboratory Press

doi:10.1101/mcs.a005751
Abstract Bloom syndrome is a rare autosomal recessive disorder with less than 300 cases reported in the literature. Bloom syndrome is characterized by chromosome instability, physical stigmata, growth deficiency, immunodeficiency, and a predisposition to cancer, most commonly leukemias, although solid tumors are reported as well. Bloom syndrome occurs in multiple ethnic groups with a higher incidence in persons of Ashkenazi Jewish origin. Few patients of Hispanic ethnicity have been reported. We report here a Mexican American family with a BLM pathogenic variant, c.2506_2507delAG, previously reported in a single patient from Mexico. In this family of four siblings, three have phenotypic features of Bloom syndrome, and BLM gene mutation was homozygous in these affected individuals. Our proband developed a rhabdomyosarcoma. Analysis of surrounding markers in the germline DNA revealed a common haplotype, suggesting a previously unrecognized founder mutation in the Hispanic population of Mexican origin.

[Supplemental material is available for this article.]

\section{INTRODUCTION}

Bloom syndrome (BS; MIM 210900) is a rare autosomal recessive disorder that clinically manifests as growth retardation, immunodeficiency, facial and cranial abnormalities, sun sensitivity, diabetes mellitus, reduced fertility, and increased cancer predisposition. This syndrome was first reported in 1954 by dermatologist Dr. David Bloom who reported development of telangiectasia in children with short stature (Bloom 1954), and knowledge of the phenotype was greatly expanded by German and Passarge (1989). The cancer phenotype observed in BS patients is broad and likely recapitulates the various malignancies present in the general population, albeit with a propensity for earlier onset and development of additional primary cancers (German 1997).

\footnotetext{
${ }^{8}$ Present address: Bristol-Myers Squibb, New York, New York 10154, USA

9Present address: Methodist Children's Hospital, San Antonio, Texas 78229, USA

${ }^{10}$ Present address: University of Virginia, Charlottesville, Virginia 22903, USA

${ }^{11}$ These authors contributed equally to this work.
} 
The BLM gene, responsible for BS in an autosomal recessive manner (Ellis et al. 1995; Hickson 2003), encodes for one of five human RecQ helicase family members (Hanada and Hickson 2007). A molecular hallmark of BS is the 10-fold increase in sister chromatic exchanges (SCEs). Because of this increase of SCEs in BS cells, BLM was hypothesized to act as an antirecombinogenic protein, suppressing spontaneous homologous recombination (HR) (Chaganti et al. 1974). In addition to increased SCEs, BS cells also have increased chromosome breaks and gaps, mutations, and micronuclei. Biochemical studies using synthetic oligonucleotide structures showed that BLM acts on specific DNA structures (Mohaghegh et al. 2001), suppresses crossing-over during HR by dissolution of these structures (Wu and Hickson 2003), and promotes branch migration (Karow et al. 2000) and annealing of complementary single-stranded DNA (Cheok et al. 2005)—all processes involved in HR. The increased incidence of cancer observed in BS patients is believed to be caused by increased chromosomal instability, as exemplified in the marked increase in SCEs and issues in appropriate chromosomal segregation.

Although Bloom syndrome has been reported in multiple races and ethnicities from around the world (German and Passarge 1989), it is most commonly reported in persons of Ashkenazi Jewish background, largely because of the presence of a founder mutation, $\mathrm{b} / \mathrm{m}^{\text {Ash }}$, a unique deletion and insertion at nucleotide 2281. Interestingly, Ellis et al. (1998) reported this same mutation in five Spanish-speaking families, not self-reporting as Jewish in the United States, Mexico, and El Salvador. It is uncertain, although possible, that these individuals descended from the Ashkenazi population many generations ago. However, Individuals of non-Ashkenazi descent with Bloom syndrome overall are reported in much smaller numbers with only two other previously reported from Hispanic individuals in a single report, one from the United States and one from Mexico (German et al. 2007).

\section{Case Report}

Our proband was a Hispanic male child, born in the United States in close proximity to the Mexican border. The patient presented at the age of $3 \mathrm{yr}, 3$ mo with a several-month history of a right-sided scrotal mass. The patient had previously been seen by a physician for failure to thrive; however, no genetic testing was performed at that time. The patient displayed short stature, failure to thrive, and developmental delay. Both height and weight were below the first percentile for age. The child was thin with notably little subcutaneous fat. Microcephaly was noted and the patient had a long thin face with coarse facial features. Multiple hyperpigmented lesions were noted in multiple body areas. He had left-sided cryptorchidism. He had normal serum glucose level and no history of diabetes.

Biopsy of the scrotal mass revealed a paratesticular embryonal rhabdomyosarcoma. The hematoxylin and eosin (H\&E)-stained sections from the biopsy material showed a variety of patterns ranging from hypercellular through moderately hypocellular areas, the latter with fibrosis. Especially noteworthy in the hypercellular areas were a mixture of small cells and spindle cells, the latter arranged in short fascicles (Fig. 1A). The cells all showed pleomorphic nuclei with hyperchromasia. Some of the smaller cells also showed very prominent eosinophilic cytoplasm highly reminiscent of rhabdomyoblasts (Fig. 1B). Tumor cells were confined to the paratesticular soft tissue without extension into testicular parenchyma and epididymis. Immunostaining showed strong cytoplasmic positivity with desmin and muscle-specific actin (Fig. 2A,B). Immunostaining with myogenin showed strong nuclear positivity; INI-1 showed retention of nuclear positivity (Fig. 2C,D).

Subsequent molecular analysis performed at St. Jude Children's Research Hospital was negative for $\mathrm{t}(2 ; 13)$ and $\mathrm{t}(1 ; 13)$ translocation, corresponding to the PAX3/FKHR and PAX7/ FKHR fusion products. 

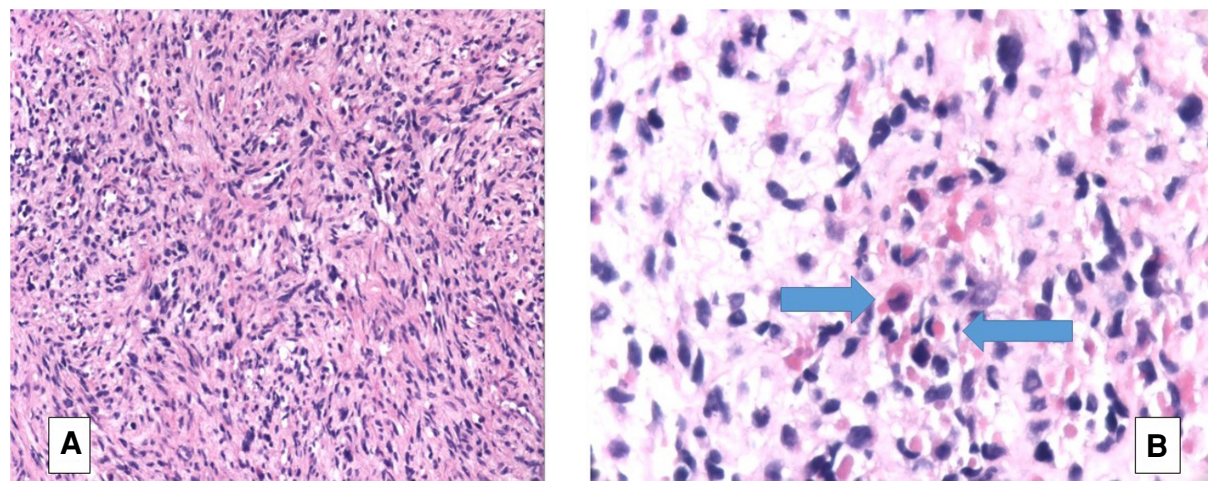

Figure 1. (A) Tumor histology: hematoxylin and eosin (H\&E) section of tumor showing a mixture of spindle and small round cells with hyperchromatic pleomorphic nuclei $(H \& E, 200 \times)$. (B) Small cells with eosinophilic cytoplasm (blue arrows) highly suggestive of rhabdomyoblasts (H\&E, 400x).

This molecular finding and the morphologic features of the tumor were consistent with a rhabdomyosarcoma of embryonal subtype.

Evaluation for metastatic disease was negative, and the tumor was determined to be in a clinical low-risk category, staged Stage I, group 2A. The patient was successfully treated with vincristine, actinomycin-D, and cyclophosphamide for 13 wk, followed by an additional 24 wk of vincristine and actinomycin-D without cyclophosphamide. Radiation therapy was not included based on favorable stage and grade. The patient had moderate thrombocytopenia expected of this type of chemotherapy, but no excessive adverse effects and no evidence of disease recurrence at $4 \mathrm{yr}$ from diagnosis. The patient had hypogammaglobulinemia noted early in his course and was supported with immunoglobulin infusions during and after his treatment. The patient is alive and without disease $>8 \mathrm{yr}$ after completion of therapy.

Prior to the diagnosis, the family had resided in South Texas on the United States-Mexico border. Both parents were born in the northern region of Mexico in proximity to the United
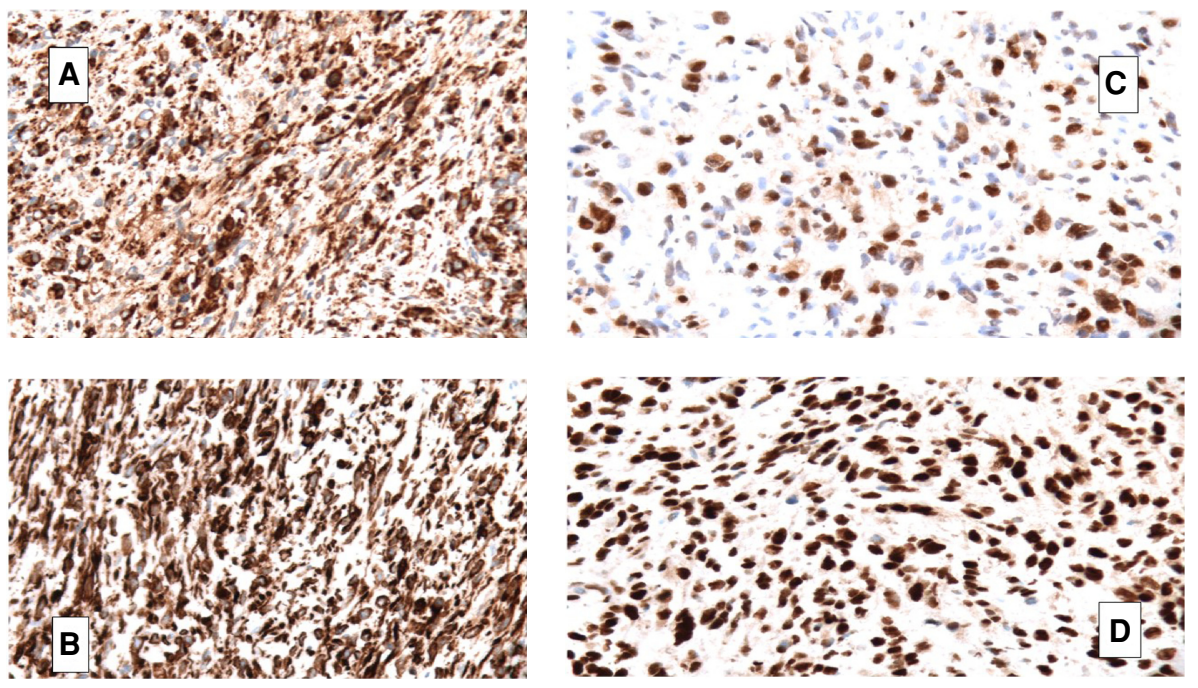

Figure 2. Immunohistochemical $(I H C)$ studies. $(A, B) I H C$ stain for desmin and muscle-specific actin, respectively, showing strong cytoplasmic positivity $(200 \times)$. (C,D) IHC stain for myogenin and INI1, respectively, demonstrating nuclear positivity (200x). 
States-Mexico border and had immigrated to the United States. The patient had three siblings: two brothers and one sister. None of the siblings had been previously evaluated for a genetic disorder, although the two male siblings were noted to have facial features similar to our patient. There was no report of consanguinity within the family. The patient, siblings, and mother were available for genetic analysis. The father was unavailable for genetic analysis.

\section{RESULTS}

A 2-bp deletion mutation in exon 12 of $B L M, c .2506 \_2507$ delAG $\left(B L M^{\text {mex }}\right)$ was observed to be homozygous in our proband and in his older and younger brothers (Table 1; Fig. 3A). His older sister and mother were heterozygous for this mutation.

The mutation status of each of the siblings was confirmed in a CLIA-certified clinical laboratory. Subsequently whole-exome analysis was performed on all available family members using the NCBI reference genome GRCh37. Analysis of the BLM locus utilized RefSeqID:NM 000057.2. Copy-number variant (CNV) analysis was carried out and confirmed that none of the available family members had other deletions of the BLM gene. Known single nucleotide polymorphisms (SNPs) flanking the BLM gene at Chr 15: 88122296-95997063 were analyzed and found to be nearly identical in the three siblings known to be homozygous for the $B L M^{\text {mex }}$ mutation (Fig. 3B).

Based on the results, the haplotype of SNPs surrounding the BLM allele inherited from the father could be imputed and we noted that both parents carried the same haplotype surrounding the $B L M^{\text {mex }}$ allele (Fig. $3 C$ ). Other regions of the genome were evaluated for potential identity by descent, but the regions were not sufficiently similar to suggest the parents were closely related. However, the presence of the identical haplotype surrounding the $B L M^{\text {mex }}$ mutation in each of the carriers and affected individuals suggests the possibility of a founder mutation that initially arose in a common ancestor of the parents. Variant properties are summarized in Table 1.

Analysis of genetic admixture confirmed similarity to the Mexican American population as compared to admixture of a known population of individuals from southern California included in the 1000 Genomes Project and known to be of Mexican background. Results of genetic admixture analysis are shown in Figure 4.

\section{DISCUSSION}

Here we report a novel case of Bloom syndrome in a Hispanic family with four children with molecular characterization of the BLM gene and surrounding region. The proband presented several phenotypic features consistent with Bloom syndrome and a paratesticular rhabdomyosarcoma. To our knowledge, this is the first reported case of rhabdomyosarcoma in a child with Bloom syndrome, although other solid tumors in childhood, including Wilms tumor, osteosarcoma, and medulloblastoma, have been reported (German 1997).

\begin{tabular}{|c|c|c|c|c|c|c|c|c|c|}
\hline Gene & $\begin{array}{l}\text { Chromo- } \\
\text { some }\end{array}$ & $\begin{array}{l}\text { HGVS DNA } \\
\text { reference }\end{array}$ & $\begin{array}{l}\text { Variant } \\
\text { type }\end{array}$ & $\begin{array}{l}\text { Predicted } \\
\text { effect }\end{array}$ & $\mathrm{dbSNP/dbVar} \mathrm{ID}$ & Genotype & ClinVar ID & $\begin{array}{c}\text { Parent of } \\
\text { origin }\end{array}$ & Comment \\
\hline BLM & 15 & NM_0000057.2 & $\begin{array}{l}\text { Patho- } \\
\text { genic }\end{array}$ & Truncation & $\begin{array}{l}\text { BLM, } \\
\quad \text { c.2506_2507delAG }\end{array}$ & Homozygous & SCV001481971 & $\begin{array}{l}\text { Mother } \\
\text { and } \\
\text { father }\end{array}$ & $\begin{array}{l}\text { Mexican } \\
\text { founder } \\
\text { mutation }\end{array}$ \\
\hline
\end{tabular}


A

I.

II.

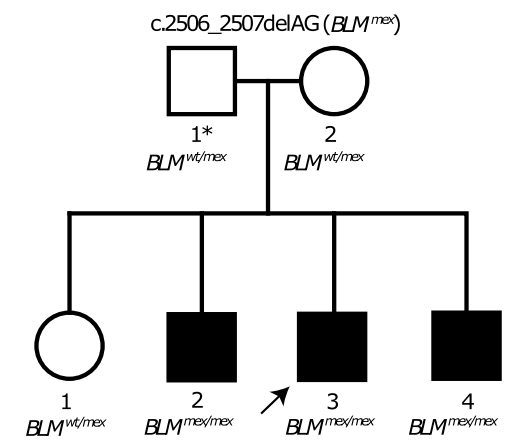

B

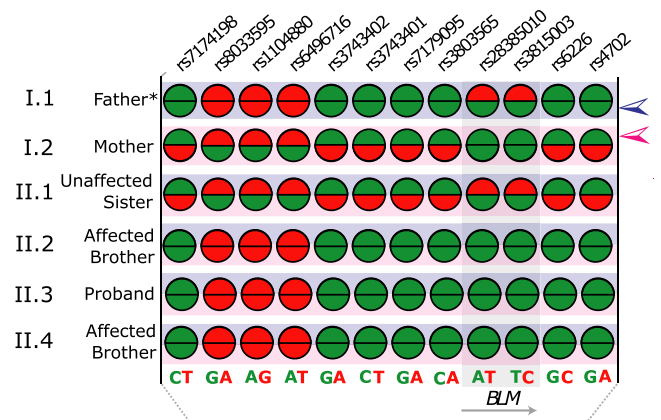

C Chr 15

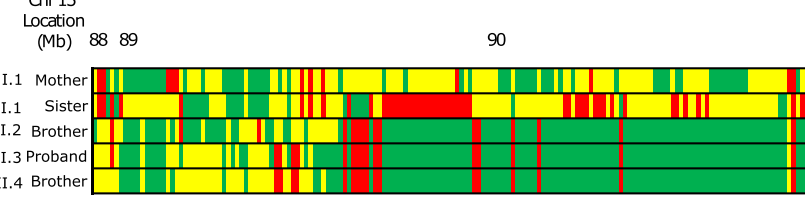

Figure 3. Sequence and haplotype analysis at BLM locus. (A) Pedigree of family carrying the BLM Mexican mutant allele (c.2506_2507delAG). Arrow indicates proband. Filled in shapes are individuals diagnosed with Bloom syndrome. The genotype, as determined by polymerase chain reaction (PCR), is listed below each individual. * indicates the genotype is inferred. (B) A closer look at the single-nucleotide polymorphisms (SNPs) closest to BLM and their potential haplotypes. Each circle represents a single SNP, and each half of the circle corresponds to the allele identity for that individual. Green indicates reference allele, and red indicates alternative allele (listed below the circles). SNPs rs28385010 and rs3815003 are within the BLM gene. Blue and pink arrowheads indicate likely paternal and maternal (respectively) BLM mutant haplotype. * indicates the genotype is inferred. (C) SNP genotyping surrounding BLM using whole-exome sequencing. Each row contains the genotype information for the indicated individual for informative SNPs (i.e., those that had complete genotypes for all five individuals and at least one individual varied from the others) flanking the BLM gene on Chromosome 15. Green indicates homozygous reference allele, red indicates homozygous alternative allele, and yellow indicates heterozygosity. SNPs that decrease within the BLM sequence are indicated by the arrow.

Sequencing of the $B L M$ gene revealed the presence of the $B L M^{\text {mex }}$ allele that was reported once before in two Hispanic individuals, one from Mexico and the other from New Mexico (German et al. 2007). In this report we document three additional patients with BS due to homozygosity of this mutant BLM allele in a family of Mexican descent living near the Texas-Mexico border and demonstrate homozygosity of flanking markers, suggesting a founder mutation.

Bloom syndrome is rare in the general population with the true incidence difficult to determine because of its overall rarity and possible underreporting, especially in distant or minority populations. Initial reports suggested that Bloom syndrome occurred more frequently in persons of Ashkenazi background. Additional analyses have reported broad genetic heterogeneity among individuals and families with Bloom syndrome; however, in a large series by German et al. it was noted that about one-third of observed mutations (19 of 64) were common in two or more individuals, suggesting possible common inheritance and thus founder mutations (German et al. 2007). Such recurring mutations were noted in families of Japanese, Italian, South American, and Spanish American as well as other European and North American heritage. A separate study from Russia reports a recurring heterozygous BLM allele (Q548X) in Slavic individuals with an adjacent additional variant, suggesting a founder allele and moreover that the variant is more common in individuals with breast cancer or a family history of breast cancer (Sokolenko et al. 2012). Most patients 
COLD SPRING HARBOR Molecular Case Studies
BLM Mexican founder mutation

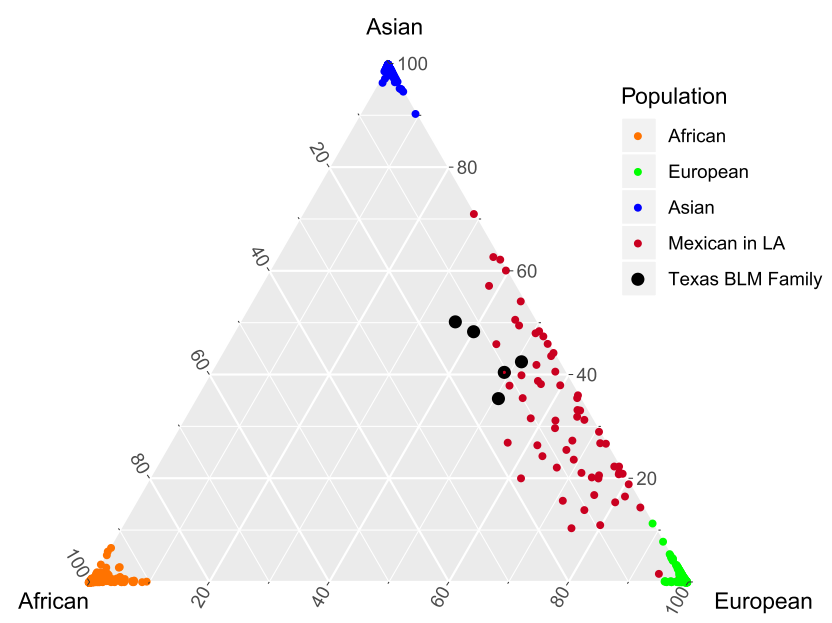

Figure 4. Triangle plot for ancestral probability for Texas family members shown by black dots based on ancestral markers from African (shown in orange), East Asian (shown in blue), and European (shown in green) populations using 1000 Genomes Project $(n=1305)$ and comparing to Mexican ancestry in Los Angeles (shown in red, $n=64$ from 1000 Genomes Project), using our unique UT-AIM250 ancestry-informative marker panel (Wang et al. 2019).

with Bloom syndrome in the non-Ashkenazi population have compound heterozygous mutations of BLM including those individuals with a recurrent mutation. Consanguinity of families with parents being related at least as second cousins were also reported in both Ashkenazi and non-Ashkenazi families, which presented homozygosity of the BLM mutation. Our finding of a homozygosity at the BLM locus in our patient is therefore notable. As the Hispanic population of Mexican descent is historically an understudied population in genetic disorders, it is possible that the allele we observe with the BLM mutation could be carried by others and could account for other undiagnosed or uncharacterized individuals with Bloom syndrome in this population. The native Mexican population is notably complex with regional genetically defined differentiation and substructure for several other variants of functional or disease significance (Ávila-Arcos 2020). Further studies will be needed to better determine the ancestral origin in terms of ancestral age and region of this reported BLM founder.

Bloom syndrome is associated with an increased risk of early-onset cancers, the most common of which is leukemia. An early report by German indicated that three of 19 individuals with Bloom syndrome developed cancers including a child with leukemia (German et al. 1965). Solid tumors in children have been reported including Wilms tumor and medulloblastomas in children and various carcinomas in adults (German 1997). We are not aware of other cases of rhabdomyosarcoma in children with Bloom syndrome; however, this may in fact be in part due to the overall rarity of rhabdomyosarcoma. It is also possible that other genetic pathogenic variants contributed to the cancer phenotype in our proband; however, none was determined.

Rhabdomyosarcoma in young children has also been associated with germline mutation of TP53 (Diller et al. 1995). It is perhaps also notable that in a mouse model heterozygous for loss of the Ptch 1 gene, additional loss of Blm has been associated with development of rhabdomyosarcoma as well as basal cell carcinoma (Davari et al. 2010). However, no germline pathogenic variant in TP53 or PTCH1 was detected in our patient (data not shown).

Screening and successful early detection of cancers in patients with Bloom syndrome is difficult; however, recent consensus recommendation from the American Association of Cancer Research Working Group in Cancer Predisposition and others have suggested avoidance of radiation, limited sun exposure, and periodic complete blood counts, screening for 
Wilms tumor with ultrasound through age 8 , and adherence to human papillomavirus (HPV) vaccination recommendations (Cunniff et al. 2017, 2018; Walsh et al. 2017). Long-term follow-up studies of additional patients or cohorts of families with Bloom syndrome will be needed to determine effectiveness of these interventions in enhancing early detection and decreasing cancer morbidity and mortality.

\section{METHODS}

Clinical suspicion of Bloom syndrome in our proband led to sequence analysis of the BLM gene in a commercial laboratory. A 2-bp deletion mutation was found in exon 12 of BLM, c.2506_2507delAG, causing a frameshift at codon 836 that would normally encode for arginine. After approval from the Institutional Review Board for further investigational genetic studies (HSC2008057H), subsequent sequence analysis of the available family members was performed at the investigators' laboratory.

To develop a polymerase chain reaction (PCR)-based diagnostic of the Mexican mutation, we analyzed the sequences of exon 12 of the human BLM gene and identified that c.2506_2507delAG results in the loss of an EcoRll site. Primers (forward: 5'-AACC CCACACTGAGTGAACGAGTCT-3' and reverse: 5'-GTAAGTTGTTGCACGTCACGT-3') were designed to amplify a 370-bp fragment of exon 12 using standard PCR reaction conditions. PCR products were visualized using a $1.0 \%$ agarose gel stained with EtBr. All confirmed amplicons underwent purification using the QIAGEN PCR cleanup kit according to manufacture protocol. Twenty-five nanograms of the product was restriction-digested with EcoRII and results visualized using a $1.5 \%$ ethidium bromide-stained agarose gel. Postdigestion, wild-type DNA will resolve into three fragments (229 bp, 83 bp, 58 bp) versus the c.2506_2507delAG variant DNA into two fragments (310 bp, 58 bp).

Upon confirmation that the EcoRll digestion from the proband's blood DNA served as a diagnostic for this specific Bloom syndrome variant, we repeated the exon 12 amplification and EcoRII digestion on DNA isolated from buccal swabs of three siblings and blood from the maternal parent of the proband. The results from the PCR assay were confirmed by subsequent whole-exome capture sequencing performed on all available family members and confirmed the variant pattern in family. In addition, the sequence variant was also confirmed with an additional blood sample in a clinical laboratory for the purpose of patient care. A summary of sequencing coverage is provided in the Supplemental File.

To confirm the reported ethnicity and ancestry, genetic admixture was determined from whole-exome data by the reported method of using a panel of exonic polymorphisms and compared to previously characterized populations (Wang et al. 2019).

\section{ADDITIONAL INFORMATION}

\section{Data Deposition and Access}

The variant has been submitted to ClinVar (https://www.ncbi.nlm.nih.gov/clinvar/) and can be found under accession number SCV001481971.

\section{Ethics Statement}

Written consent for genetic research was obtained from the parent on behalf of the minor children according to the Institutional Review Board of the University of Texas Health Science Center at San Antonio (HSC2008057H). 
Competing Interest Statement The authors have declared no competing interest.

Received August 15, 2020; accepted in revised form February 8, 2021.

\section{Acknowledgments}

We acknowledge the family reported who kindly allowed us to study the four children so as to advance knowledge.

\section{Funding}

Funding was provided by grant T32AG021890 (to A.D.B.), a Hyundai Hope on Wheels grant (to A.J.R.B. and G.T.), IIMS-GCCRI Ambassador Award Pilot funds and grant K22ES012264 (to A.J.R.B.), a Greehey Family Foundation Fellowship (to E.H.S.), grant CPRIT PP120089 (to G.T.), a Cancer Center Support Grant, National Institutes of Health (NIH) grant 5P30CA054174-21 (to G.T., A.J.R.B., and Y.C.), and the CPRIT Core Facility grant RP160732 (to Y.C.).

\section{REFERENCES}

Ávila-Arcos MC, McManus KF, Sandoval K, Rodríguez-Rodríguez JE, Villa-Islas V, Martin AR, Luisi P, PenalozaEspinosa RI, Eng C, Huntsman S, et al. 2020. Population history and gene divergence in native Mexicans inferred from 76 human exomes. Mol Biol Evol 37: 994-1006. doi:10.1093/molbev/msz282

Bloom D. 1954. Congenital telangiectatic erythema resembling lupus erythematosus in dwarfs; probably a syndrome entity. AMA Am J Dis Child 88: 754-758. doi:10.1001/archpedi.1954.02050100756008

Chaganti RS, Schonberg S, German J. 1974. A manyfold increase in sister chromatid exchanges in Bloom's syndrome lymphocytes. Proc Natl Acad Sci 71: 4508-4512. doi:10.1073/pnas.71.11.4508

Cheok CF, Wu L, Garcia PL, Janscak P, Hickson ID. 2005. The Bloom's syndrome helicase promotes the annealing of complementary single-stranded DNA. Nucleic Acids Res 33: 3932-3941. doi:10.1093/nar/gki712

Cunniff C, Bassetti JA, Ellis NA. 2017. Bloom's syndrome: clinical spectrum, molecular pathogenesis, and cancer predisposition. Mol Syndromol 8: 4-23. doi:10.1159/000452082

Cunniff C, Djavid AR, Carrubba S, Cohen B, Ellis NA, Levy CF, Jeong S, Lederman HM, Vogiatzi M, Walsh MF, et al. 2018. Health supervision for people with Bloom syndrome. Am J Med Genet 176: 1872-1881. doi:10 .1002/ajmg.a.40374

Davari P, Hebert JL, Albertson DG, Huey B, Roy R, Mancianti ML, Horvai AE, McDoniel LD, Schultz RA, Epstein EH. 2010. Loss of Blm enhances basal cell carcinoma and rhabdomyosarcoma tumorigenesis in Ptch $1^{+/-}$ mice. Carcinogenesis 31: 968-973. doi:10.1093/carcin/bgp309

Diller L, Sexsmith E, Gottlieb A, Li FP, Malkin D. 1995. Germline p53 mutations are frequently detected in young children with rhabdomyosarcoma. J Clin Invest 95: 1606-1611. doi:10.1172/JCl117834

Ellis NA, Groden J, Ye TZ, Straughen J, Lennon DJ, Ciocci S, Proytcheva M, German J. 1995. The Bloom's syndrome gene product is homologous to RecQ helicases. Cell 83: 655-666. doi:10.1016/0092-8674(95) 90105-1

Ellis NA, Ciocci S, Proytcheva M, Lennon D, Groden J, German J. 1998. The Ashkenazic Jewish Bloom syndrome mutation blm ${ }^{A s h}$ is present in non-Jewish Americans of Spanish ancestry. Am J Hum Genet 63: 1685-1693. doi:10.1086/302167

German J. 1997. Bloom's syndrome. XX. The first 100 cancers. Cancer Genet Cytogenet 93: 100-106. doi:10 $.1016 / \mathrm{s} 0165-4608(96) 00336-6$

German J, Passarge E. 1989. Bloom's syndrome. XII. Report from the Registry for 1987. Clin Genet 35: 57-69. doi:10.1111/j.1399-0004.1989.tb02905.x

German J, Archibald R, Bloom D. 1965. Chromosomal breakage in a rare and probably genetically determined syndrome of man. Science 148: 506-507. doi:10.1126/science.148.3669.506

German J, Sanz MM, Ciocci S, Ye TZ, Ellis NA. 2007. Syndrome-causing mutations of the BLM gene in persons in the Bloom's Syndrome Registry. Hum Mutat 28: 743-753. doi:10.1002/humu.20501

Hanada K, Hickson ID. 2007. Molecular genetics of RecQ helicase disorders. Cell Mol Life Sci 64: 2306-2322. doi:10.1007/s00018-007-7121-z

Hickson ID. 2003. RecQ helicases: caretakers of the genome. Nat Rev Cancer 3: 169-178. doi:10.1038/ $\operatorname{nrc1012}$

Karow JK, Constantinou A, Li JL, West SC, Hickson ID. 2000. The Bloom's syndrome gene product promotes branch migration of Holliday junctions. Proc Natl Acad Sci 97: 6504-6508. doi:10.1073/pnas .100448097

Mohaghegh P, Karow JK, Brosh RM, Bohr VA, Hickson ID. 2001. The Bloom's and Werner's syndrome proteins are DNA structure-specific helicases. Nucleic Acids Res 29: 2843-2849. doi:10.1093/nar/29.13 .2843 
Sokolenko AP, lyevleva AG, Preobrazhenskaya EV, Mitiushkina NV, Abysheva SN, Suspitsin EN, Kuligna ES, Gorochova TV, Pfeiffer W, Togo AV, et al. 2012. High prevalence and breast cancer predisposing role of the BLM c.1642 C>T (Q548X) mutation in Russia. Int J Cancer 130: 2867-2873. doi:10.1002/ijc .26342

Walsh MF, Chang VY, Kohlmann WK, Scott HS, Cunniff C, Bourdeaut F, Molenaar JJ, Porter CC, Sandlund JT, Plon SE, et al. 2017. Recommendations for childhood cancer screening and surveillance in DNA repair disorders. Clin Cancer Res 23: e23-e31. doi:10.1158/1078-0432.CCR-17-0465

Wang LJ, Zhang CW, Su SC, Che HH, Chiu YC, Lai Z, Bouamar H, Ramirez AG, Cigarroa FG, Sun LZ, et al. 2019. An ancestry informative marker panel design for individual ancestry estimation of Hispanic population using whole exome sequencing data. BMC Genomics 20: 1007. doi:10.1186/s12864-019-6333-6

Wu L, Hickson ID. 2003. The Bloom's syndrome helicase suppresses crossing over during homologous recombination. Nature 426: 870-874. doi:10.1038/nature02253 


\title{
COLD SPRING HARBOR Molecular Case Studies
}

\section{Bloom syndrome in a Mexican American family with rhabdomyosarcoma: evidence of a Mexican founder mutation}

\author{
Erin H. Sybouts, Adam D. Brown, Maria G. Falcon-Cantrill, et al.
}

Cold Spring Harb Mol Case Stud 2021, 7: a005751

Access the most recent version at doi: $10.1101 / \mathrm{mcs} . a 005751$
Supplementary http://molecularcasestudies.cshlp.org/content/suppl/2021/04/06/mcs.a005751.D Material C1
References This article cites 22 articles, 4 of which can be accessed free at: http://molecularcasestudies.cshlp.org/content/7/2/a005751.full.html\#ref-list-1
License This article is distributed under the terms of the Creative Commons Attribution-NonCommercial License, which permits reuse and redistribution, except for commercial purposes, provided that the original author and source are credited.
Email Alerting Receive free email alerts when new articles cite this article - sign up in the box at the Service top right corner of the article or click here.

